\title{
Antitumor effect of lapatinib and cytotoxic agents by suppression of E2F1 in HER2-positive breast cancer
}

\author{
AKIKO MATSUMOTO $^{1,2}$, TETSU HAYASHIDA ${ }^{1}$, MAIKO TAKAHASHI ${ }^{1}$, \\ HIROMITSU JINNO $^{2}$ and YUKO KITAGAWA ${ }^{1}$ \\ ${ }^{1}$ Department of Surgery, Keio University School of Medicine, Tokyo 160-8582; \\ ${ }^{2}$ Department of Surgery, Teikyo University School of Medicine, Tokyo 173-8606, Japan
}

Received November 27, 2017; Accepted March 26, 2018

DOI: $10.3892 / \mathrm{mmr} .2018 .9068$

\begin{abstract}
The human epidermal growth factor receptor 2 (HER2)-targeting agent, lapatinib, combined with oral fluoropyrimidine capecitabine, has been previously demonstrated to be an effective treatment option for patients with trastuzumab-resistant HER2-positive metastatic breast cancer. To investigate the molecular mechanisms associated with the interactions between lapatinib and capecitabine, the effect of treatment with lapatinib and phosphatidylinositol-4,5-bisphosphate 3-kinase (PI3K) inhibitors on the expression of E2F transcription factor 1 (E2F1) and thymidylate synthase (TS), which is associated with an increased response to 5-fluorouracil (5-FU)-based chemotherapy, was determined in HER2-positive breast cancer cells. The results of reverse transcription-quantitative polymerase chain reaction demonstrated that administration of lapatinib and PI3K inhibitors decreased the mRNA expression of TS and E2F1, a transcription factor that promotes TS gene expression, in SKBR3 and T47D cell lines. Furthermore, treatment with lapatinib and PI3K inhibitors also suppressed the mRNA expression of ribonucleotide reductase M1 subunit (RRM1), an important determinant of gemcitabine resistance, and DNA topoisomerase II- $\alpha$ (TOP2A), a molecular target of anthracyclines, in SKBR3 and T47D cell lines. Western blot analysis further demonstrated that the phosphorylation of Akt was inhibited by lapatinib, and the results of the MTT assay revealed that the combination of lapatinib with either 5-FU or gemcitabine demonstrated synergistic antitumor effects, whereas a combinatory treatment of lapatinib with epirubicin, a typical anthracycline drug, exhibited antagonistic antitumor effects in HER2-positive breast cancer cells. These results indicate that the synergistic antitumor effects exhibited by combinatory
\end{abstract}

Correspondence to: Dr Tetsu Hayashida, Department of Surgery, Keio University School of Medicine, 35 Shinanomachi, Shinjuku, Tokyo 160-8582, Japan

E-mail: tetsu@keio.jp

Key words: breast cancer, human epidermal growth factor receptor 2, lapatinib, capecitabine, gemcitabine, E2F transcription factor 1 treatment of lapatinib with capecitabine may be induced via the suppression of E2F1-mediated TS expression.

\section{Introduction}

Breast cancer is a major health issue affecting women worldwide, with $\sim 1.4$ million novel diagnoses and $>450,000$ mortalities occurring annually (1). Overexpression of human epidermal growth factor receptor 2 (HER2) has been reported in $\sim 20 \%$ of patients with breast cancer and is associated with aggressive clinical behavior and poor prognosis (2). HER2-targeting agents, including the anti-HER2 monoclonal antibody trastuzumab, have been demonstrated to improve the overall survival rate of patients with HER2-positive breast cancer (3). However, resistance to trastuzumab may eventually develop and, in certain cases, relapse may occur following adjuvant therapy.

Lapatinib is a small-molecule tyrosine kinase inhibitor that suppresses the expression of epidermal growth factor receptor (EGFR) and HER2, as well as the activity of the downstream phosphatidylinositol-4,5-bisphosphate 3-kinase (PI3K)/Akt pathway, which have important roles in cell proliferation and survival (4). Administration of lapatinib in combination with the oral fluoropyrimidine capecitabine has been previously demonstrated to be an effective treatment option for trastuzumab-resistant HER2-positive metastatic breast cancer (5). This combination treatment was approved by the United States Food and Drug Administration and European Medicines Agency based on evidence from a phase III study that demonstrated an increased time to progression (TTP) in patients treated with lapatinib in combination with capecitabine, as compared with capecitabine alone (6). Capecitabine was designed to preferentially generate 5-fluorouracil (5-FU) in tumor tissue. Correlations have been previously demonstrated between decreased thymidylate synthase (TS) expression and a higher response rate to 5-FU-based chemotherapy $(7,8)$. In gastric cancer, lapatinib induces the downregulation of E2F transcription factor 1 (E2F1), which enhances the transcription of the TS gene (9). Therefore, we hypothesized that lapatinib may downregulate TS expression in HER2-positive breast cancer cells via downregulation of E2F1 expression.

The efficacy of combination therapy involving lapatinib and other cytotoxic agents in HER2-positive breast cancer 
remains undetermined. E2F1 regulates the expression of ribonucleotide reductase catalytic M1 subunit (RRM1), an important determinant of gemcitabine resistance (10), and DNA topoisomerase II- $\alpha$ (TOP2A), a molecular target of anthracyclines (11). Therefore, we further hypothesized that lapatinib may exhibit a synergistic antitumor effect in combination with not only capecitabine, but also gemcitabine, and it may also exhibit an antagonistic effect when used in combination with anthracyclines.

In the present study, the molecular mechanisms associated with the synergistic antitumor effects observed following treatment with lapatinib and capecitabine were investigated, as well as the efficacy of interactions between lapatinib and other cytotoxic agents for the treatment of patients with breast cancer exhibiting overexpression of cellular HER2.

\section{Materials and methods}

Cell lines and cell culture. The SKBR3 HER2-overexpressing human breast cancer cell line and the T47D human breast cancer cell line with moderate expression of HER2 $(12,13)$ were obtained from the American Type Culture Collection (Manassas, VA,USA). SKBR3 cells were cultured in Dulbecco's modified Eagle's medium supplemented with $10 \%$ fetal bovine serum (FBS; Gibco; Thermo Fisher Scientific Inc., Waltham, MA, USA) and 5\% $0.1 \mathrm{mM}$ penicillin-streptomycin. T47D cells were cultured in RPMI-1640 medium (Gibco; Thermo Fisher Scientific Inc.) supplemented with $10 \%$ FBS and 5\% $0.1 \mathrm{mM}$ penicillin-streptomycin. All cell lines were incubated at $37^{\circ} \mathrm{C}$ in $95 \%$ humidified atmosphere containing $5 \% \mathrm{CO}_{2}$.

Reverse transcription-quantitative polymerase chain reaction (RT-qPCR). SKBR3 and T47D cells $\left(3 \times 10^{5}\right.$ cells/well) were seeded in 6-well plates and were serum-starved at $37^{\circ} \mathrm{C}$ for $24 \mathrm{~h}$. Cells were treated with epidermal growth factor (EGF; $100 \mathrm{ng} / \mathrm{ml}$; Gibco; Thermo Fisher Scientific Inc.) at $37^{\circ} \mathrm{C}$ for $30 \mathrm{~min}$ and subsequently treated with dimethyl sulfoxide (DMSO; Sigma-Aldrich; Merck KGaA, Darmstadt, Germany) vehicle control, LY294002 (10 $\mu \mathrm{M}$; Sigma-Aldrich; Merck KGaA), wortmannin (200 nM; Sigma-Aldrich; Merck KGaA) or lapatinib (1 $\mu \mathrm{M}$; Selleck Chemicals, Houston, TX, USA) at $37^{\circ} \mathrm{C}$ for $48 \mathrm{~h}$. Total RNA was extracted from the cells using an RNeasy Plus Mini kit (Qiagen, Inc., Valencia, CA, USA) and first-strand cDNA was synthesized using the PrimeScript RT reagent kit (Takara Bio, Inc., Otsu, Japan) to investigate the expression levels of genes of interest. The protocol for cDNA synthesis was $37^{\circ} \mathrm{C}$ for $15 \mathrm{~min}$, followed by $85^{\circ} \mathrm{C}$ for 5 sec. qPCR analysis was performed using Power SYBR Green Master Mix (Applied Biosystems; Thermo Fisher Scientific, Inc.) on a 7300 Real-Time PCR system (Applied Biosystems; Thermo Fisher Scientific, Inc.). The following program was run: Predenaturing for $10 \mathrm{~min}$ at $95^{\circ} \mathrm{C}$, amplification for 40 cycles $\left(15 \mathrm{sec}\right.$ of denaturation at $95^{\circ} \mathrm{C}, 1 \mathrm{~min}$ of annealing/extension at $60^{\circ} \mathrm{C}$ ). GAPDH was used as the internal control and the $2^{-\triangle \Delta \mathrm{Cq}}$ method (14) was used to determine protein expression levels. The sequences of all primers used in the present study were as follows: TOP2A, 5'-ACC AGCACATCAAAGGAAGC-3' (forward) and 5'-AATCCT CAGGAAGCCCAAGT-3' (reverse); TS, 5'-GCCTCGGTG TGCCTTTCA-3' (forward) and 5'-CCCGTGATGTGCGCA
AT-3' (reverse); RRM1, 5'-ACTAAGCACCCTGACTATGCT ATCC-3' (forward) and 5'-CTTCCATCACATCACTGAACA CTTT-3' (reverse); E2F1, 5'-CAAGAAGTCCAAGAACCA CATCC-3' (forward) and 5'-AGATATTCATCAGGTGGT CCAGC-3' (reverse); and GAPDH, ATCATCCCTGCCTCT ACTGG-3' (forward) and 5'-TTTCTAGACGGCAGGTCA GGT-3' (reverse). The experiment was performed three times.

Western blot analysis. SKBR3 and T47D cells were serum-starved at $37^{\circ} \mathrm{C}$ for $24 \mathrm{~h}$ prior to treatment with EGF $(100 \mathrm{ng} / \mathrm{ml})$ at $37^{\circ} \mathrm{C}$ for $30 \mathrm{~min}$. Cells were subsequently washed and treated with DMSO vehicle control, LY294002 (40 $\mu \mathrm{M})$, wortmannin $(500 \mathrm{nM})$ or lapatinib $(1 \mu \mathrm{M})$ at $37^{\circ} \mathrm{C}$ for $30 \mathrm{~min}, 24$ or 48 h. Cells were lysed using a ReadyPrep Protein Extraction kit (Bio-Rad Laboratories, Inc., Hercules, CA, USA) supplemented with a protease inhibitor cocktail (Roche Diagnostics, Basel, Switzerland) and phosphatase inhibitor cocktail (Roche Diagnostics), according to the manufacturer's protocol. Protein samples were quantified by the Bradford method and total protein (60 $\mu \mathrm{g}$ per lane) was fractionated on 4-15\%, 10-well comb Mini-PROTEAN TGX gels (Bio-Rad Laboratories, Inc.) and electrophoretically transferred onto TransBlot Turbo Mini polyvinylidene fluoride membranes (Bio-Rad Laboratories, Inc.). Membranes were subsequently blocked with Amersham ECL Blocking Agent (GE Healthcare Life Sciences, Little Chalfont, UK) in Tris-buffered saline and $0.1 \%$ Tween for 30-60 min at room temperature. Following this, membranes were exposed to the following primary antibodies overnight at $4^{\circ} \mathrm{C}$ : Anti-Akt (mouse; 1:800; cat. no. 2920S; Cell Signaling Technology, Inc., Danvers, MA, USA), anti-phosphorylated-Akt (p-Akt; rabbit; 1:800; cat. no. 4060S; Cell Signaling Technology, Inc.), anti-E2F1 (mouse; 1:1,000; cat. no. sc-251; Santa Cruz Biotechnology, Inc., Dallas, TX, USA), anti-lamin (mouse; 1:800; cat. no. ab8980; Abcam, Cambridge, UK) and anti-GAPDH (mouse; 1:2,000; cat. no. sc-47724; Santa Cruz Biotechnology, Inc.). Membranes were subsequently exposed to the horseradish peroxidase (HRP)-conjugated anti-rabbit (1:2,000; cat. no. NA934V; GE Healthcare Life Sciences) and anti-mouse (1:1,000; cat. no. NA931V; GE Healthcare Life Sciences) secondary antibodies at room temperature for $1 \mathrm{~h}$. Proteins were visualized using Luminata Forte Western HRP substrate (EMD Millipore, Billerica, MA, USA).

MTT cell proliferation assay. An MTT assay was performed to investigate cell proliferation. SKBR3 and T47D cells were plated in 96-well plates at a density of $1 \times 10^{4}$ cells/well and cultured at $37^{\circ} \mathrm{C}$ for $24 \mathrm{~h}$. Following this, cells were treated with various concentrations of lapatinib, 5-FU (Sigma-Aldrich; Merck KGaA), gemcitabine (Sigma-Aldrich; Merck KGaA) and epirubicin (Sigma-Aldrich; Merck KGaA) at $37^{\circ} \mathrm{C}$ for 5 days. The concentrations of each drug was as follows: 5-FU and lapatinib for T47D were $0.1,0.2,1,2,10$ and $20 \mu \mathrm{M}$, and $0.05,0.1,0.5,1,5$ and $10 \mu \mathrm{M}$, respectively; 5-FU and lapatinib for SKBR3 were $0.5,1,5,10,50$ and $100 \mu \mathrm{M}$, and 0.25, 0.5, 2.5, 5, 25 and $50 \mu \mathrm{M}$, respectively; gemcitabine and lapatinib for T47D were $0.01,0.1,0.5,1,5$ and $10 \mu \mathrm{M}$, and $0.005,0.05,0.25$, $0.5,2.5$ and $5 \mu \mathrm{M}$, respectively; gemcitabine and lapatinib for SKBR3 were $0.05,0.1,1,5,10$ and $100 \mu \mathrm{M}$, and 0.025, 0.05, $0.5,2.5,5$ and $50 \mu \mathrm{M}$, respectively; epirubicin and lapatinib for T47D were $0.01,0.1,0.5,1,5$ and $10 \mu \mathrm{M}$; epirubicin and 

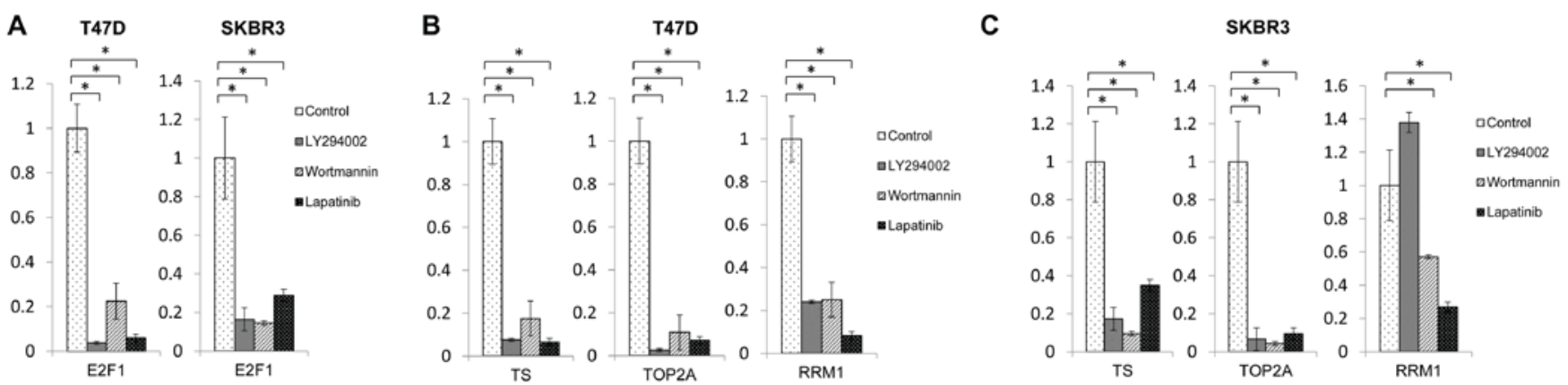

Figure 1. Expression levels of E2F1, TS, TOP2A and RRM1 mRNAs following treatment with lapatinib and phosphatidylinositol-4,5-bisphosphate 3-kinase inhibitors. (A) E2F1 expression levels following treatment with lapatinib, LY294002 and wortmannin in T47D and SKBR3 cells. TS, TOP2A and RRM1 expression levels following treatment with lapatinib, LY294002 and wortmannin in (B) T47D cells and (C) SKBR3 cells. "P<0.05, as indicated. E2F1, E2F transcription factor 1; TS, thymidylate synthase; TOP2A, DNA topoisomerase II- $\alpha$; RRM1, ribonucleotide reductase M1 subunit.

lapatinib for SKBR3 were 0.005, 0.025, 0.1, 1, 10 and $100 \mu \mathrm{M}$. MTT $(10 \mu l)$ was subsequently added to each well and the cells were incubated at $37^{\circ} \mathrm{C}$ for a further $3 \mathrm{~h}$. Following incubation, DMSO $(>99 \% ; 130 \mu \mathrm{l})$ was added to each well and the absorbance at $540 \mathrm{~nm}$ was determined using a SUNRISE Rainbow RC-R (Tecan Group, Ltd., Mannedorf, Switzerland). The antitumor effect of the different combinations of lapatinib with various cytotoxic drugs (5-FU, gemcitabine and epirubicin) was analyzed according to a previously described method (15). Interactions between two drugs (lapatinib and 5-FU, lapatinib and gemcitabine, and lapatinib and epirubicin) were estimated via the combination index (CI) using CalcuSyn software (version 2; Biosoft, Cambridge, UK). CI $<1, \mathrm{CI}=1$ and $\mathrm{CI}>1$ scores revealed synergistic, additive and antagonistic effects, respectively.

Statistical analysis. Statistical analysis was performed using SPSS statistical software (version 19; IBM Corp., Armonk, NY, USA). RT-qPCR was performed in triplicate and the mRNA expression (mean \pm standard deviation) was determined using one-way analysis of variance followed by Games Howell post-hoc test. $\mathrm{P}<0.05$ was considered to indicate a statistically significant difference.

\section{Results}

E2F1, TS, TOP2A and RRM1 expression levels are attenuated following treatment with lapatinib and PI3K inhibitors. To investigate the molecular mechanisms associated with interactions between lapatinib and capecitabine, the effect of treatment with lapatinib and PI3K inhibitors on E2F1 and TS expression in HER2-positive breast cancer cells was investigated. The results of RT-qPCR demonstrated that treatment with lapatinib and PI3K inhibitors decreased the mRNA expression of E2F1 and TS in T47D and SKBR3 cells at $48 \mathrm{~h}$ post-treatment (Fig. 1A-C). Decreased TS expression has been reported to be associated with a higher response rate to 5-FU-based chemotherapy $(7,8)$, and the E2F1 transcription factor promotes TS expression (16). Therefore, these results indicate that downregulation of E2F1 expression by lapatinib may induce TS downregulation, which subsequently enhances the effect of capecitabine. Furthermore, treatment with lapatinib and PI3K inhibitors also downregulated the expression of
TOP2A (Fig. 1B and C), a molecular target of anthracyclines, in both cell lines. In addition, treatment with lapatinib and PI3K inhibitors suppressed RRM1 expression, an important determinant of gemcitabine resistance, in T47D cells (Fig. 1B). In SKBR3 cells, RRM1 downregulation was demonstrated following treatment with wortmannin and lapatinib; however, RRM1 expression was enhanced in SKBR3 cells following treatment with LY294002 (Fig. 1C), indicating different responses to different PI3K inhibitors.

Treatment with lapatinib and PI3K inhibitors downregulates E2F1 protein expression via the PI3K/Akt pathway. To investigate the effects of lapatinib treatment on the PI3K/Akt signaling pathway, levels of p-Akt and E2F1 were investigated using western blot analysis. Following treatment with PI3K inhibitors and lapatinib for $30 \mathrm{~min}$, levels of p-Akt were revealed to be inhibited in both T47D and SKBR3 cells (Fig. 2A). Western blot analysis also revealed that E2F1 protein expression was downregulated following treatment with PI3K inhibitors and lapatinib for $24 \mathrm{~h}$ in T47D cells (Fig. 2B). In SKBR3 cells, it was revealed that E2F1 protein levels were suppressed by treatment with lapatinib for $48 \mathrm{~h}$ (Fig. 2C); however, these levels were unaffected by treatment with PI3K inhibitors or lapatinib for $24 \mathrm{~h}$ (data not shown). Following $48 \mathrm{~h}$ of treatment with PI3K inhibitors, it was not possible to determine the expression levels of E2F1 in SKBR3 cells, as the majority of the cells were died unexpectedly (Fig. 2B). These results indicate that E2F1 downregulation following treatment with lapatinib may be a result of inhibition of the PI3K/Akt signaling pathway.

Antitumor effect of combination therapy using lapatinib and cytotoxic agents. An MTT assay was performed to investigate the cell proliferative effects of combination therapy involving lapatinib and various cytotoxic agents (5-FU, gemcitabine and epirubicin) on HER2-positive breast cancer cells. Capecitabine is designed to preferentially generate 5-FU in tumor tissue. Therefore, 5-FU was used instead of capecitabine for the MTT assay. The results of the MTT assay revealed that cell viability was markedly decreased in T47D and SKBR3 cells following treatment with 5-FU or lapatinib alone at various concentrations, and the inhibitory effect was enhanced when 5 -FU was administered in combination with lapatinib in a 
A

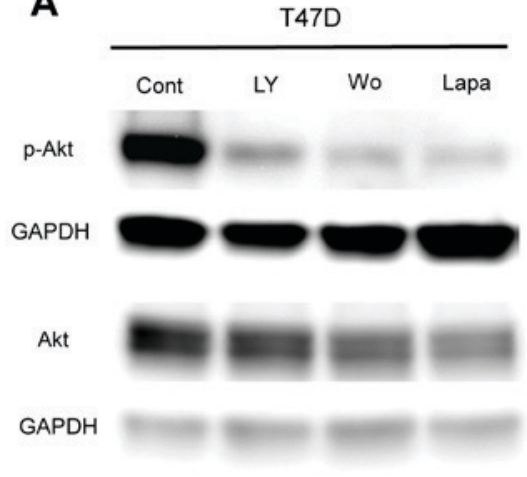

B

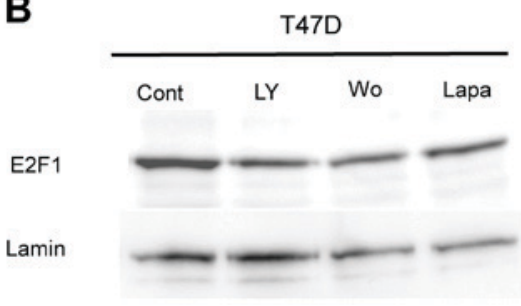

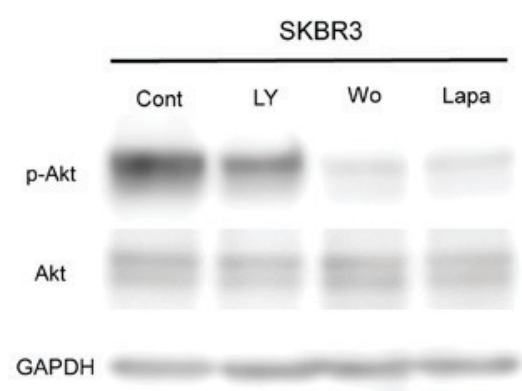

C

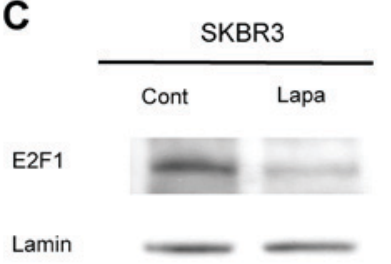

Figure 2. Effect of treatment with lapatinib and phosphatidylinositol-4,5-bisphosphate 3-kinase inhibitors on levels of Akt phosphorylation and E2F1 protein expression in T47D and SKBR3 cells. (A) Levels of Akt phosphorylation were suppressed in T47D and SKBR3 cells following treatment with lapatinib, LY294002 and wortmannin for $30 \mathrm{~min}$. (B) Expression levels of E2F1 in T47D cells were suppressed following treatment with lapatinib, LY294002 and wortmannin for $24 \mathrm{~h}$. (C) E2F1 expression levels were suppressed in SKBR3 cells following 48 h treatment with lapatinib. E2F1 protein expression in LY294002- and wortmannin-treated SKBR3 cells at this time-point could not be measured as the majority of cells were dead. E2F1, E2F transcription factor 1; Cont, control; LY, LY294002; Wo, wortmannin; Lapa, lapatinib; p-Akt, phosphorylated-Akt.

dose-dependent manner (Fig. 3A). Similar inhibitory effects were also observed in both cell lines following treatment with gemcitabine and lapatinib (Fig. 3B), whereas combinatory treatment with epirubicin (an anthracycline drug) and lapatinib did not markedly suppress cell proliferation compared with that observed following treatment with epirubicin or lapatinib alone (Fig. 3C). In addition, the interaction between two drugs based on CI values was investigated. A synergistic antitumor effect $(\mathrm{CI}<1.0)$ on cell proliferation was demonstrated when T47D and SKBR3 cells were treated with a combination of 5-FU and lapatinib at each concentration (Fig. 3D). A similar synergistic effect involving combined treatment of gemcitabine and lapatinib was also observed in both cell lines (Fig. 3E). By contrast, the combination of epirubicin and lapatinib demonstrated antagonistic effects $(\mathrm{CI}>1.0)$ at each concentration (Fig. 3F). These results indicate that combination treatment with lapatinib and either 5-FU or gemcitabine exhibited synergistic antitumor effects, whereas lapatinib treatment in combination with epirubicin exhibited antagonistic antitumor effects in HER2-positive breast cancer cells.

Overall, the results of the present study indicate that downregulation of TS, RRM1 and TOP2A in HER2-positive breast cancer cells following lapatinib treatment is attributable to E2F1 downregulation, which may be a result of inhibition of the PI3K/Akt pathway, a process which is summarized in Fig. 4.

\section{Discussion}

The results of the present study demonstrated that combinatory treatment with lapatinib and 5-FU, a capecitabine metabolite, induced a synergistic antitumor effect on HER2-positive breast cancer cells. In addition, RT-qPCR and western blot analyses revealed that treatment of cells with lapatinib or PI3K inhibitors downregulated E2F1 and TS expression. These results indicated that inhibition of the PI3K/Akt signaling pathway may contribute to the downregulation of E2F1 and TS expression by lapatinib. These results are consistent with those reported by Tanizaki et al (9), who demonstrated that in gastric cancer cells exhibiting overexpression of HER2, treatment with lapatinib downregulated TS expression via the PI3K/Akt signaling pathway, which may be a result of downregulated E2F1 expression.

Lapatinib is a dualEGFR andHER2 tyrosine kinase inhibitor approved by the United States Food and Drug Administration for the treatment of patients with HER2-positive metastatic or locally advanced breast cancer. In a phase III trial of combined treatment with lapatinib and capecitabine vs. treatment with capecitabine alone in patients with HER2-positive advanced breast cancer (6), the combinatory treatment significantly improved TTP compared with capecitabine treatment alone (6.2 vs. 4.3 months; hazard ratio, 0.57 ; $\mathrm{P}<0.001$ ). Despite the development of novel and effective molecular-targeted therapies, such as pertuzumab (17) and trastuzumab emtansine (18), lapatinib continues to represent an important treatment option for patients with trastuzumab-resistant HER2-positive metastatic breast cancer. Therefore, the results of the present study concerning the molecular mechanism associated with the synergistic antitumor effects of combination treatment with lapatinib and capecitabine provide important insight into effective options for treatment of HER2-positive breast cancer.

The efficacy of interactions between lapatinib and other cytotoxic agents, such as gemcitabine and epirubicin, was also investigated with regards to HER2-positive breast cancer 
A

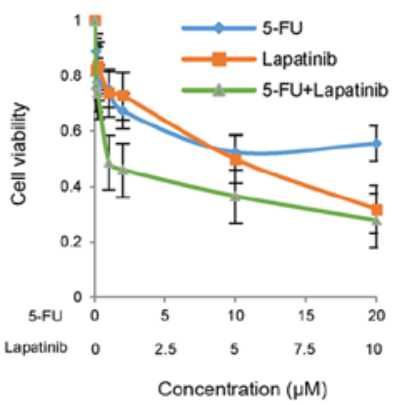

T47D

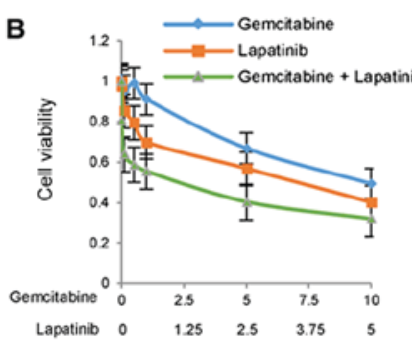

Concentration $(\mu \mathrm{M})$

T47D

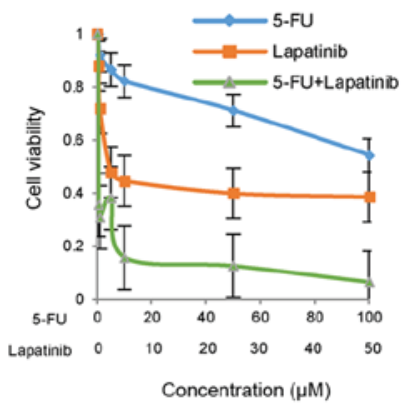

SKBR3

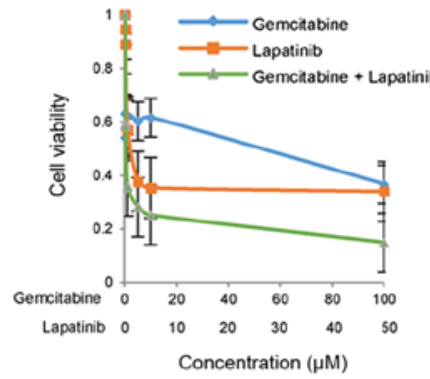

SKBR3
D

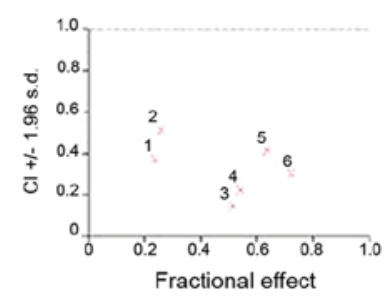

T47D
5-FU + Lapatinib

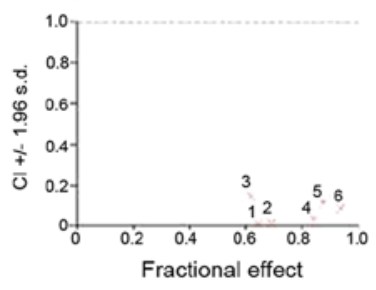

SKBR3

E

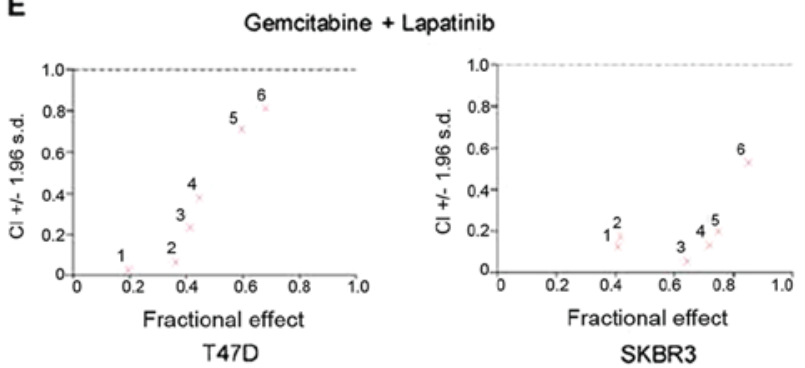

$\mathbf{F}$

Epirubicin + Lapatinib

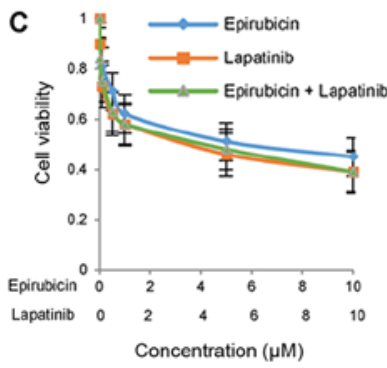

T47D

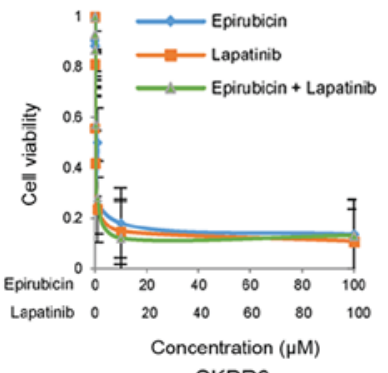

SKBR3

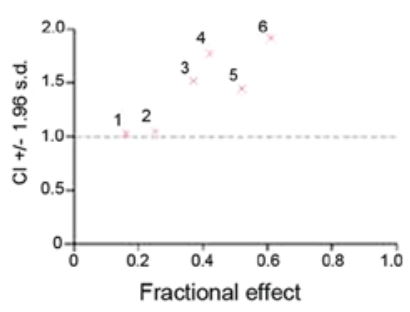

T47D

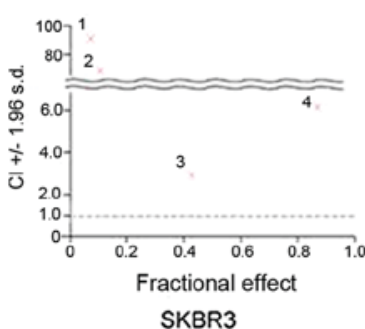

SKBR3

Figure 3. Antitumor effects of combinatory treatments of lapatinib with various cytotoxic agents. Effects of combination treatment consisting of (A) lapatinib with 5-FU, (B) lapatinib with gemcitabine and (C) lapatinib with epirubicin on T47D and SKBR3 cell proliferation, compared with individual treatments. CI analysis indicated that combination of lapatinib with (D) 5-FU and (E) gemcitabine led to synergistic antitumor effects, while combination of lapatinib with (F) epirubicin led to an antagonistic antitumor effect. CI $<1.0$ was considered to indicate a synergistic antitumor effect, while CI $>1.0$ represents an antagonistic antitumor effect. 5-FU, 5-fluorouracil; CI, combination index.

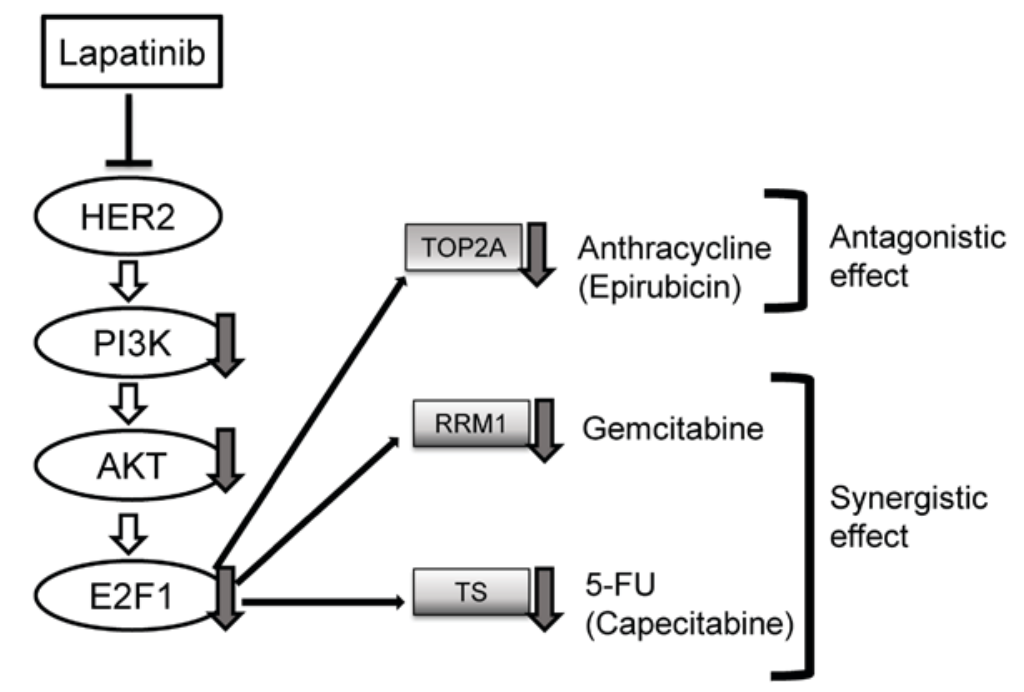

Figure 4. Hypothesized molecular mechanism associated with the antitumor effects in T47D and SKBR3 cells following combinatory treatment of lapatinib with 5-FU (capecitabine), gemcitabine or anthracyclines (epirubicin). HER2, human epidermal growth factor receptor 2; PI3K, phosphatidylinositol-4,5-bisphosphate 3-kinase; E2F1, E2F transcription factor 1; TOP2A, DNA topoisomerase II- $\alpha$; RRM1, ribonucleotide reductase M1 subunit; TS, thymidylate synthase; 5-FU, 5-fluorouracil. 
treatment. The results revealed that treatment with lapatinib or PI3K inhibitors markedly downregulated the expression of RRM1, an important determinant of gemcitabine resistance, as well as TOP2A, a molecular target of anthracyclines such as epirubicin. Activation of the PI3K/Akt signaling pathway has been associated with upregulated E2F1 expression (19,20). E2F1 is involved in the regulation of RRM1 (10) and TOP2A (11) expression. Therefore, the results of the present study indicate that lapatinib may synergistically interact with gemcitabine, resulting in a combinatorial antagonistic effect with epirubicin and leading to the downregulation of E2F1 and RRM1, or the downregulation of TOP2A, via the PI3K/Akt pathway.

In a phase III trial, combination therapy involving lapatinib and capecitabine frequently resulted in adverse side effects, such as diarrhea, hand-foot syndrome, and nausea, which were reported in 60,49 , and $44 \%$ of the patients, respectively (6). By contrast, gemcitabine administered to patients with metastatic breast cancer in previous phase II trials rarely exhibited symptomatic toxicities, such as gastrointestinal toxicity and hand-foot syndrome (21). Therefore, combination treatment with lapatinib and gemcitabine may represent a more effective treatment option for patients with HER2-positive metastatic breast cancer. The results of the present study regarding epirubicin treatment, a member of the anthracycline class of drugs, indicate that it was ineffective in combination with lapatinib. Furthermore, the combination of anti-HER2 therapy, including lapatinib, with anthracycline has not been recommended in clinical practice, as it has been revealed to be associated with high incidences of cardiac toxicity (22).

In conclusion, the present study investigated the molecular mechanisms associated with the combined treatment of lapatinib and capecitabine in HER2-positive breast cancer cells, and determined the potential efficacy of interactions between lapatinib and gemcitabine, as well as the effect of lapatinib and epirubicin. The results of the present study lead to the suggestion that a clinical trial of combination chemotherapy using lapatinib and gemcitabine for the treatment of breast cancer exhibiting an overexpression of HER2 may be promising.

\section{Acknowledgements}

The authors would like to thank Mr. H. Okazaki, Mr. K. Miyao and Dr Y. Nakazawa (Keio University School of Medicine, Tokyo, Japan) for their technical support.

\section{Funding}

The current study was supported by the Ministry of Education, Culture, Sports, Science and Technology (Tokyo, Japan) Grant-in-Aid for Young Scientists (grant no. JP25861163).

\section{Availability of data and materials}

The datasets used and/or analyzed during the current study are available from the corresponding author on reasonable request.

\section{Authors' contributions}

TH, MT, HJ and YK conceived and designed the experiments. $\mathrm{AM}$ and TH performed the experiments. AM and TH analyzed the data. AM wrote the manuscript. AM, TH, MT, HJ and YK discussed the results and revised the manuscript.

\section{Ethics approval and consent to participate}

Not applicable.

\section{Consent for publication}

Not applicable.

\section{Competing interests}

The authors declare that they have no competing interests.

\section{References}

1. Siegel R, Naishadham D and Jemal A: Cancer statistics, 2012. CA Cancer J Clin 62: 10-29, 2012.

2. Slamon DJ, Clark GM, Wong SG, Levin WJ, Ullrich A and McGuire WL: Human breast cancer: Correlation of relapse and survival with amplification of the HER-2/neu oncogene. Science 235: 177-182, 1987.

3. Slamon DJ, Leyland-Jones B, Shak S, Fuchs H, Paton V, Bajamonde A, Fleming T, Eiermann W, Wolter J, Pegram M, et al: Use of chemotherapy plus a monoclonal antibody against HER2 for metastatic breast cancer that overexpresses HER2. N Engl J Med 344: 783-792, 2001.

4. Medina PJ and Goodin S: Lapatinib: A dual inhibitor of human epidermal growth factor receptor tyrosine kinases. Clin Ther 30: 1426-1447, 2008

5. Blackwell KL, Kaplan EH, Franco SX, Marcom PK, Maleski JE, Sorensen MJ and Berger MS: A phase II, open-label, multicenter study of GW572016 in patients with trastuzumab-refractory metastatic breast cancer. Proc Ann Meeting Am Soc Clin Oncol 23: 196, 2004.

6. Geyer CE, Forster J, Lindquist D, Chan S, Romieu CG Pienkowski T, Jagiello-Gruszfeld A, Crown J, Chan A, Kaufman B, et al: Lapatinib plus capecitabine for HER2-positive advanced breast cancer. N Engl J Med 355: 2733-2743, 2006.

7. Johnston PG, Drake JC, Trepel J and Allegra CJ: Immunological quantitation of thymidylate synthase using the monoclonal antibody TS 106 in 5-fluorouracil-sensitive and -resistant human cancer cell lines. Cancer Res 52: 4306-4312, 1992.

8. Johnston PG, Fisher ER, Rockette HE, Fisher B, Wolmark N, Drake JC, Chabner BA and Allegra CJ: The role of thymidylate synthase expression in prognosis and outcome of adjuvant chemotherapy in patients with rectal cancer. J Clin Oncol 12: 2640-2647, 1994.

9. Tanizaki J, Okamoto I, Takezawa K, Tsukioka S, Uchida J, Kiniwa M, Fukuoka M and Nakagawa K: Synergistic antitumor effect of S-1 and HER2-targeting agents in gastric cancer with HER2 amplification. Mol Cancer Ther 9: 1198-1207, 2010.

10. Yasui K, Okamoto $\mathrm{H}$, Arii $\mathrm{S}$ and Inazawa $\mathrm{J}$ : Association of over-expressed TFDP1 with progression of hepatocellular carcinomas. J Hum Genet 48: 609-613, 2003.

11. Nakajima T, Yasui K, Zen K, Inagaki Y, Fujii H, Minami M, Tanaka S, Taniwaki M, Itoh Y, Arii S, et al: Activation of B-Myb by E2F1 in hepatocellular carcinoma. Hepatol Res 38: 886-895, 2008.

12. Gschwantler-Kaulich D, Grunt TW, Muhr D, Wagner R, Kölbl H and Singer CF: HER Specific TKIs exert their antineoplastic effects on breast cancer cell lines through the involvement of STAT5 and JNK. PLoS One 11: e0146311, 2016.

13. Ludyga N, Anastasov N, Gonzalez-Vasconcellos I, Ram M, Höfler H and Aubele M: Impact of protein tyrosine kinase 6 (PTK6) on human epidermal growth factor receptor (HER) signalling in breast cancer. Mol Biosyst 7: 1603-1612, 2011.

14. Livak KJ and Schmittgen TD: Analysis of relative gene expression data using real-time quantitative PCR and the 2(-Delta Delta C(T)) method. Methods 25: 402-408, 2001.

15. Chou TC and Talalay P: Quantitative analysis of dose-effect relationships: The combined effects of multiple drugs or enzyme inhibitors. Adv Enzyme Regul 22: 27-55, 1984. 
16. DeGregori J, Kowalik T and Nevins JR: Cellular targets for activation by the E2F1 transcription factor include DNA synthesis- and G1/S-regulatory genes. Mol Cell Biol 15: 4215-4224, 1995.

17. Baselga J, Cortés J, Kim SB, Im SA, Hegg R, Im YH, Roman L, Pedrini JL, Pienkowski T, Knott A, et al: Pertuzumab plus trastuzumab plus docetaxel for metastatic breast cancer. N Engl J Med 366: 109-119, 2012.

18. Hurvitz SA, Dirix L, Kocsis J, Bianchi GV, Lu J, Vinholes J, Guardino E, Song C, Tong B, Ng V, et al: Phase II randomized study of trastuzumab emtansine versus trastuzumab plus docetaxel in patients with human epidermal growth factor receptor 2-positive metastatic breast cancer. J Clin Oncol 31: $1157-1163,2013$

19. Hallstrom TC and Nevins JR: Specificity in the activation and control of transcription factor E2F-dependent apoptosis. Proc Natl Acad Sci USA 100: 10848-10853, 2003.
20. Liu K, Paik JC, Wang B, Lin FT and Lin WC: Regulation of TopBP1 oligomerization by Akt/PKB for cell survival. EMBO J 25: 4795-4807, 2006.

21. Blackstein M, Vogel CL, Ambinder R, Cowan J, Iglesias J and Melemed A: Gemcitabine as first-line therapy in patients with metastatic breast cancer: A phase II trial. Oncology 62: 2-8, 2002.

22. Curigliano G, Cardinale D, Suter T, Plataniotis G, de Azambuja E, Sandri MT, Criscitiello C, Goldhirsch A, Cipolla C and Roila F; ESMO Guidelines Working Group: Cardiovascular toxicity induced by chemotherapy, targeted agents and radiotherapy: ESMO clinical practice guidelines. Ann Oncol 23 (Suppl 7): vii155-vii166, 2012. 